\title{
"Rigid body rotation" of the left ventricle in hypoplastic right-heart syndrome: a case from the three-dimensional speckle-tracking echocardiographic MAGYAR-Path Study
}

\author{
Attila Nemes, Kálmán Havasi, Tamás Forster \\ 2nd Department of Medicine and Cardiology Centre, Medical Faculty, Albert Szent-Györgyi Clinical Center, \\ University of Szeged, Szeged, Hungary
}

\begin{abstract}
Left ventricular twist results from the movement of two orthogonally oriented muscular bands of the helical myocardial structure, with a consequent clockwise rotation of the left ventricular base and counterclockwise rotation of the left ventricular apex. To the best of the authors' knowledge, this is the first time that left ventricular "rigid body rotation", the near absence of left ventricular twist in hypoplastic right-heart syndrome, has been demonstrated.
\end{abstract}

Keywords: Hypoplastic right-heart syndrome; three-dimensional; echocardiography; speckle-tracking; left ventricular rotation

Received: 6 November 2013; Accepted: 9 May 2014; First published online: 16 June 2014

L EFT VENTRICULAR TWIST RESULTS FROM THE MOVEment of two orthogonally oriented muscular bands of the helical myocardial structure, with a consequent clockwise rotation of the left ventricular base and counterclockwise rotation of the left ventricular apex. ${ }^{1,2} \mathrm{~A}$ three-dimensional speckle-tracking echocardiography seems to be a feasible method for the non-invasive evaluation of left ventricular rotational
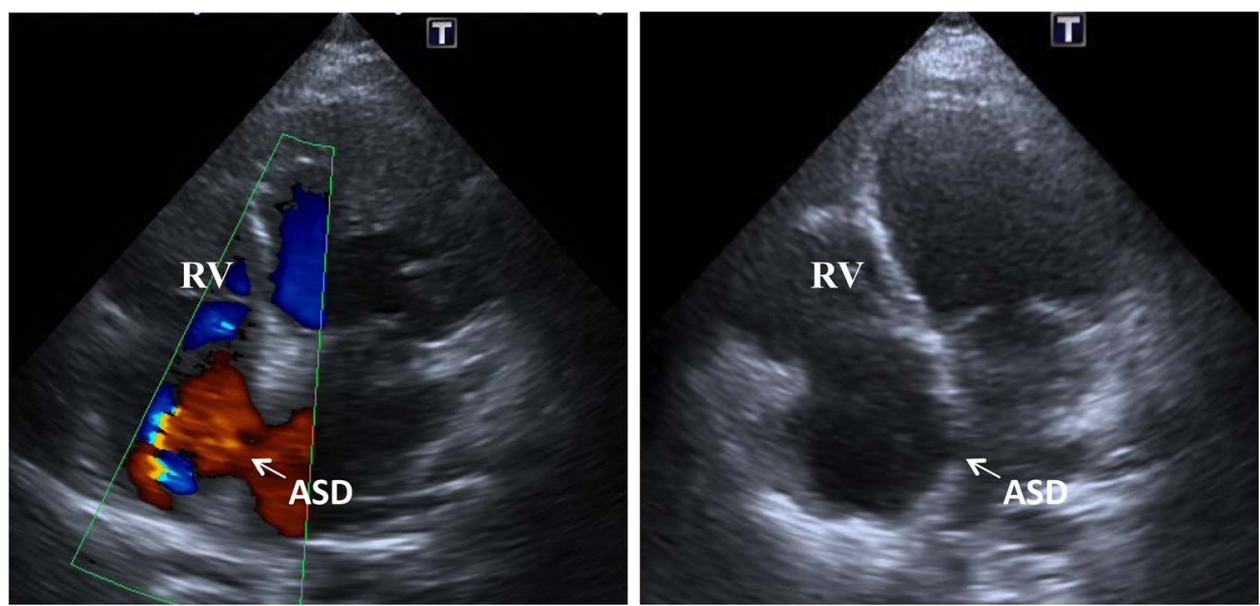

Figure 1.

Transthoracic Doppler echocardiography images from a 19-year-old girl with palliated bypoplastic right-heart syndrome. The images demonstrate an intact ventricular septum, atrial septal defect (ASD), and small right ventricular $(R V)$ cavity.

Correspondence to: A. Nemes, MD, PhD, FESC, 2nd Department of Medicine and Cardiology Center, Medical Faculty, University of Szeged, Korányi fasor 6, P.O. Box 427, H-6720 Szeged, Hungary. Tel: 366254 5220; Fax: 366254 4568; E-mail: nemes.attila@med.u-szeged.hu 


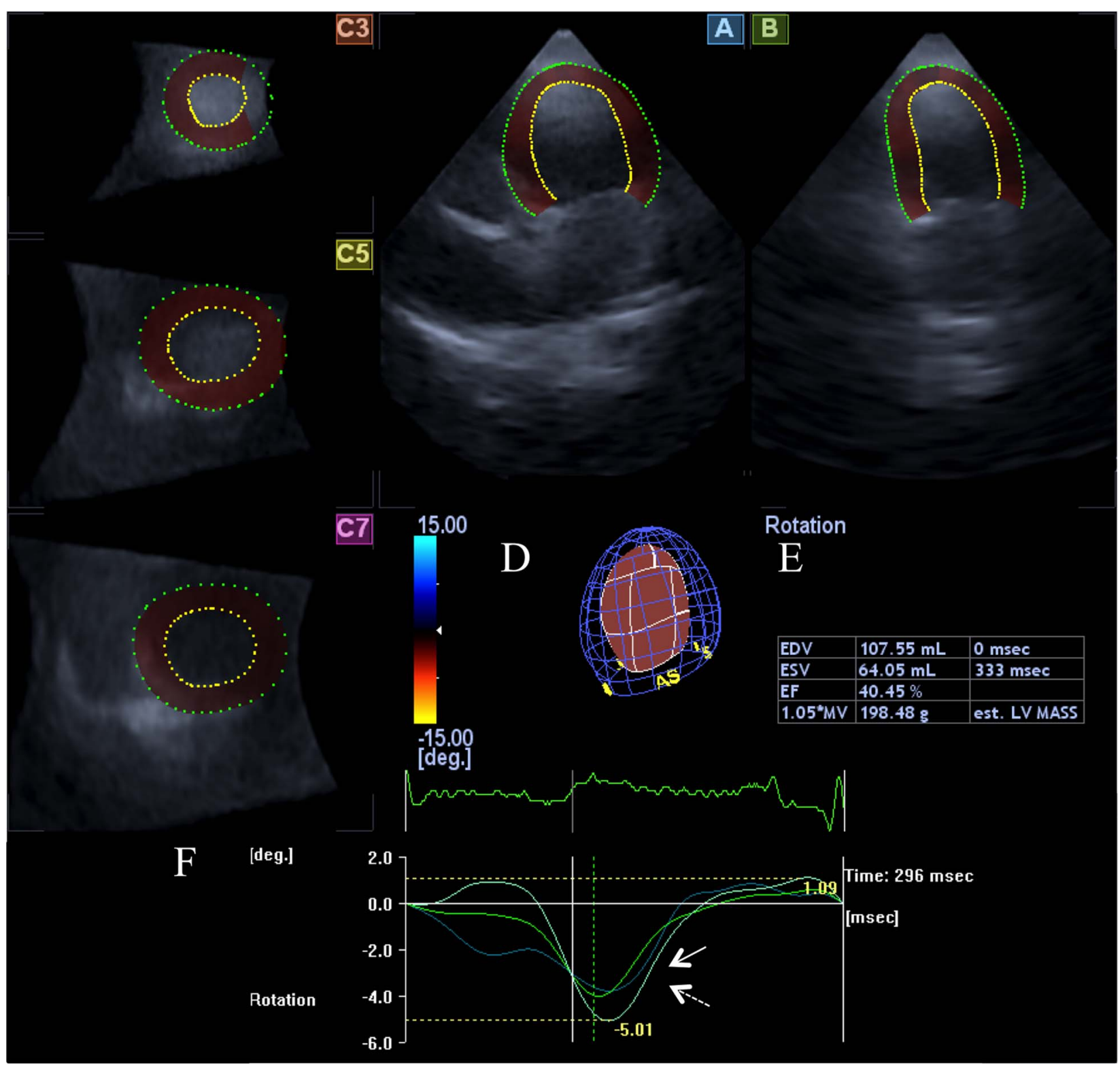

Figure 2.

In a patient with palliated hypoplastic right-heart syndrome, apical four-chamber $(\boldsymbol{A})$ and two-chamber $(\boldsymbol{B})$ views and short-axis views $(\boldsymbol{C} 3$, C5, C7) at different levels of the left ventricle extracted from the three-dimensional echocardiographic data set are presented. A threedimensional cast of the left ventricle $(\boldsymbol{D})$ and calculated volumetric and functional left ventricular parameters are also demonstrated $(\boldsymbol{E})$. Left ventricular basal and apical rotations proved to be in the same direction with near absence of left ventricular twist $(\boldsymbol{F})$. EDV $=$ end-diastolic volume, $E F=$ ejection, ESV $=$ end-systolic volume.

mechanics. ${ }^{3,4}$ This case report presents alterations in left ventricular twist in a patient with palliated hypoplastic right-heart syndrome.

\section{Case report}

A 19-year-old girl with surgically palliated hypoplastic right-heart syndrome with intact ventricular septum, ostium secundum atrial septal defect, and small right ventricular cavity is presented. The patient had undergone palliation with a superior caval vein to pulmonary artery shunt - bidirectional Glenn - at 3 years of age. The patient proved to be compensated without palpitations. She was involved in the MAGYAR-Path Study - Motion Analysis of the heart and Great vessels bY three-dimensionAl speckle-tRacking echocardiography in Pathological cases. A complete two-dimensional Doppler echocardiography was carried out with a commercially available Toshiba Artida ${ }^{\mathrm{TM}}$ (Toshiba Medical Systems, Tokyo, Japan) echocardiography equipment with a PST-30SBP (1-5 MHz) phased-array transducer 


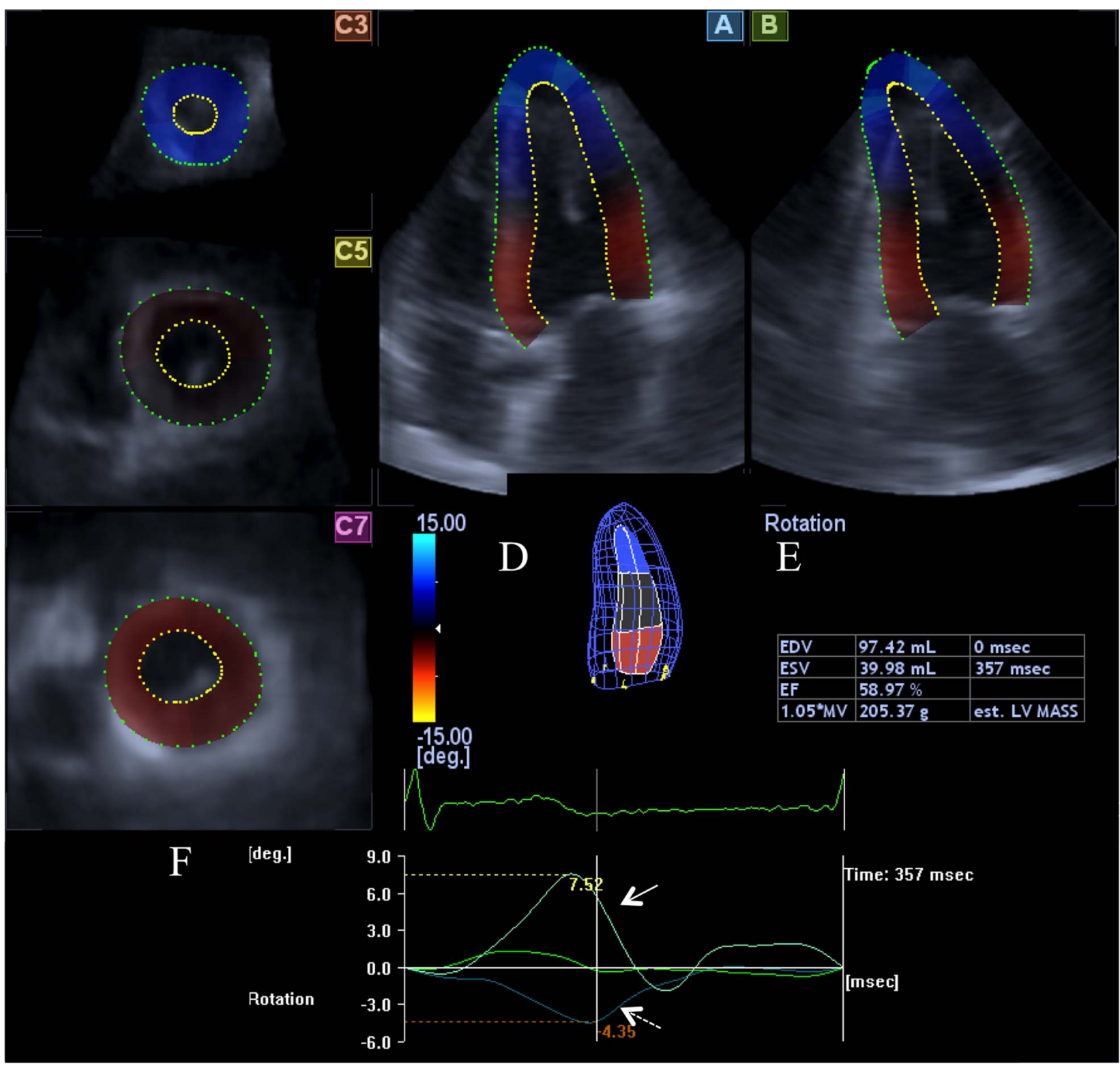

Figure 3.

In a bealthy patient, apical four-chamber $(\boldsymbol{A})$ and two-chamber $(\boldsymbol{B})$ views and short-axis views $(\boldsymbol{C} 3, \boldsymbol{C} 5, \boldsymbol{C} 7)$ at different levels of the left ventricle extracted from the three-dimensional echocardiographic data set are presented. A three-dimensional cast of the left ventricle (D) and calculated volumetric and functional left ventricular parameters are also demonstrated (E). Counterclockwise rotation of the left ventricular apex (white arrow, positive value) and clockwise rotation of the left ventricular base (dashed arrow, negative value) are shown demonstrating normal rotational directions $(\boldsymbol{F}) . E D V=$ end-diastolic volume, $E F=$ ejection, $E S V=$ end-systolic volume.

(Toshiba Medical Systems, Tokyo, Japan), confirming right ventricular dysplasia and atrial septal defect (Fig 1). The movement of the septum was found to be dyskinetic. Following two-dimensional echocardiographic study, three-dimensional data acquisition was performed using a PST-25SX matrix-array transducer (Toshiba Medical Systems) with threedimensional speckle-tracking echocardiographic capability. During a constant RR interval and within a single breath-hold, six wedge-shaped subvolumes were acquired from an apical window to create a full-volume three-dimensional data set. The chamber quantification was performed off-line using 3D Wall Motion Tracking software version 2.5 (Toshiba Medical Systems). The multiple long- (A, B) and short-axis views (C3, C5, C7) extracted from threedimensional echocardiographic data sets of the patient with hypoplastic right-heart syndrome and that of a healthy control are demonstrated in Figures 2 and 3 . Visual information on left ventricular rotational mechanics are shown in colour overlay superimposed on grey-scale images in both cases. 
Left ventricular end-diastolic and end-systolic volumes, ejection fraction, mass and right ventricular outflow tract, and tricuspid annular plane systolic excursion of the patient proved to be $107 \mathrm{ml}$, $64 \mathrm{ml}, 40 \%, 198 \mathrm{~g}, 17 \mathrm{~mm}$, and $14 \mathrm{~mm}$, respectively. Results of the present case with hypoplastic right-heart syndrome suggest that all left ventricular regions move in almost the same clockwise direction (Fig 2f, dashed arrow, negative value). Global left ventricular radial, circumferential, longitudinal, three-dimensional and area strain parameters, and the range for all segments proved to be $5.1 \pm 4.3$, $-12.2 \pm 8.0,-14.1 \pm 8.5,6.0 \pm 4.8$, and $-24.3 \pm$ $13.8 \%$, respectively. Quantitative data of left ventricular rotation of the control patient demonstrate adequate rotational directions with counterclockwise motion of the left ventricular apex (white arrow, positive value) and clockwise motion of the left ventricular base (dashed arrow, negative value; Fig 3F).

\section{Discussion}

To the best of the authors' knowledge, this is the first time that left ventricular basal and apical rotations could be in the same clockwise direction, with near absence of left ventricular twist as called "rigid body rotation" of the left ventricle in hypoplastic rightheart syndrome, has been demonstrated. In a recent study using two-dimensional speckle-tracking echocardiography, left ventricular "rigid body rotation" was demonstrated in patients with non-compaction cardiomyopathy. ${ }^{5}$ The near absence of left ventricular twist was found to be an objective, quantitative, and reproducible functional criterion with good predictive value for the diagnosis of left ventricular non-compaction. 5 This phenomenon was found in 53-100\% of non-compaction cardiomyopathy patients by other working groups. ${ }^{7,8}$ Similar alterations in left ventricular rotational mechanism could be demonstrated in a patient with univentricular heart with left ventricular characteristics as well. ${ }^{9}$ Hypoplastic right-heart syndrome is a congenital heart defect in which the right ventricle of the heart fails to develop appropriately. However, rotational mechanics of the left ventricle has never been assessed by speckle-tracking echocardiography in this disease.

It is known that two-dimensional speckle-tracking echocardiography based on frame-by-frame tracking of speckle patterns created by interference of the ultrasound beam within the myocardial tissue in one plane is likely to be affected by geometric assumptions and the use of foreshortened apical views. ${ }^{4-6}$ The threedimensional speckle-tracking echocardiography detects speckles in the three-dimensional space, and therefore is able to merge benefits of three-dimensional and speckletracking echocardiographies, allowing clinicians to visualise the heart as it is: a three-dimensional organ. ${ }^{4}$ It was found to be a quantitave way to assess left ventricular rotation and twist deformations., $3,4,8-10$ "Rigid body rotation" of the left ventricle was confirmed by this methodology in a non-compaction cardiomyopathy, as well. 8,10

In conclusion, left ventricular "rigid body rotation" was identified in a single patient with hypoplastic right-heart syndrome. This finding could be partially explained by the impairment in ventricle-to-ventricle interaction. Alterations in the anatomic myocardial fibre orientation, which is a left-handed helix in the epicardium and a right-handed helix in the endocardium in normal situation, could be altered in this disease. However, other reasons could not be excluded as well. Further studies with larger number of hypoplastic right-heart syndrome patients are warranted to confirm our findings with deeper insights into pathophysiology.

\section{Acknowledgement}

Many thanks to linguistic corrections by Dr Péter Hausinger.

\section{Financial Support}

Attila Nemes, MD, holds a János Bolyai Research Fellowship (Budapest, Hungary).

\section{Conflicts of Interest}

None.

\section{Ethical Standards}

The authors assert that all procedures contributing to this work comply with the ethical standards of the relevant national guidelines on human experimentation and with the Helsinki Declaration of 1975, as revised in 2008, and has been approved by the institutional committee of the University of Szeged.

\section{References}

1. Sengupta PP, Tajik AJ, Chandrasekaran K, Khandheria BK. Twist mechanics of the left ventricle: principles and application. JACC Cardiovasc Imaging 2008; 1: 366-376.

2. Nemes A, Kalapos A, Domsik P, Forster T. Left ventricular rotation and twist of the heart. Clarification of some concepts. Orv Hetil 2012; 153: 1547-1551.

3. Zhou Z, Ashraf M, Hu D, et al. Three-dimensional speckle-tracking imaging for left ventricular rotation measurement: an in vitro validation study. J Ultrasound Med 2010; 29: 903-909.

4. Nemes A, Kalapos A, Domsik P, Forster T. Three-dimensional speckle-tracking echocardiography - a further step in non-invasive three-dimensional cardiac imaging. Orv Hetil 2012; 153: 1570-1577.

5. Van Dalen BM, Caliskan K, Soliman OI, et al. Left ventricular solid body rotation in non-compaction cardiomyopathy: a potential new 
objective and quantitative functional diagnostic criterion? Eur J Heart Fail 2008; 10: 1088-1093.

6. Van Dalen BM, Caliskan K, Soliman OI, et al. Diagnostic value of rigid body rotation in noncompaction cardiomyopathy. J Am Soc Echocardiogr 2011; 24: 548-555.

7. Peters F, Khandheria BK, Libhaber E, et al. Left ventricular twist in left ventricular noncompaction. Eur Heart J Cardiovasc Imaging 2014; 15: 48-55.

8. Kalapos A, Domsik P, Forster T, Nemes A. Comparative evaluation of left ventricular function by two-dimensional echocardiography and three-dimensional speckle-tracking echocardiography in noncompaction cardiomyopathy. Results from the MAGYAR-Path Study. Orv Hetil 2013; 154: 1352-1359.

9. Nemes A, Havasi K, Domsik P, Forster T. Can univentricular heart be associated with "rigid body rotation"? - A case from the threedimensional speckle-tracking echocardiographic MAGYARPath Study. Hellenic J Cardiol (in press).

10. Nemes A, Kalapos A, Domsik P, Forster T. Identification of left ventricular "rigid body rotation" by three-dimensional speckletracking echocardiography in a patient with noncompaction of the left ventricle: a case from the MAGYAR-Path Study. Echocardiography 2012; 29: E237-E240. 